\title{
Impact of Many-Body Effects on Landau Levels in Graphene
}

\author{
J. Sonntag, ${ }_{1,2, *}$ S. Reichardt, ${ }^{1,3}$ L. Wirtz, ${ }^{3}$ B. Beschoten, ${ }^{1}$ M. I. Katsnelson, ${ }^{4}$ F. Libisch, ${ }^{5}$ and C. Stampfer ${ }^{1,2}$ \\ ${ }^{1}$ JARA-FIT and 2nd Institute of Physics, RWTH Aachen University, 52074 Aachen, Germany \\ ${ }^{2}$ Peter Grünberg Institute (PGI-9), Forschungszentrum Jülich, 52425 Jülich, Germany \\ ${ }^{3}$ Physics and Materials Science Research Unit, University of Luxembourg, L-1511 Luxembourg, Luxembourg \\ ${ }^{4}$ Institute for Molecules and Materials, Radboud University, 6525AJ Nijmegen, Netherlands \\ ${ }^{5}$ Institute for Theoretical Physics, Vienna University of Technology, 1040 Vienna, Austria
}

(Received 29 November 2017; published 4 May 2018)

\begin{abstract}
We present magneto-Raman spectroscopy measurements on suspended graphene to investigate the charge carrier density-dependent electron-electron interaction in the presence of Landau levels. Utilizing gate-tunable magnetophonon resonances, we extract the charge carrier density dependence of the Landau level transition energies and the associated effective Fermi velocity $v_{F}$. In contrast to the logarithmic divergence of $v_{F}$ at zero magnetic field, we find a piecewise linear scaling of $v_{F}$ as a function of the charge carrier density, due to a magnetic-field-induced suppression of the long-range Coulomb interaction. We quantitatively confirm our experimental findings by performing tight-binding calculations on the level of the Hartree-Fock approximation, which also allow us to estimate an excitonic binding energy of $\approx 6 \mathrm{meV}$ contained in the experimentally extracted Landau level transitions energies.
\end{abstract}

DOI: 10.1103/PhysRevLett.120.187701

Many-body interactions crucially influence the electronic properties of graphene [1]. They are essential to the understanding of such effects as unconventional quantum Hall states [2,3], graphene plasmonics [4-6], or the formation of a viscous Dirac fermion liquid $[7,8]$. In particular, the long-range electron-electron interaction is predicted to heavily modify the single-particle band structure close to the charge neutrality point (CNP), which is described by a logarithmically divergent effective Fermi velocity $v_{F}$ [9-12]. This charge carrier density $\left(n_{\mathrm{el}}\right)$ dependent band structure renormalization at low or vanishing magnetic fields was experimentally confirmed with various different experimental techniques such as transport measurements [13], quantum capacitance measurements [14], angle-resolved photoemission spectroscopy (ARPES) $[15,16]$, and scanning tunneling spectroscopy $[17,18]$. Still, very little is known about the effects of electron-electron interaction on the band structure of graphene in the presence of quantizing magnetic fields, i.e., Landau levels (LLs). The only experimental [19] and theoretical [20] studies so far focused on the scaling of the effective Fermi velocity with magnetic field $B$ at a fixed charge carrier density close to the CNP. Interestingly, the extracted $v_{F}$ is not in agreement with earlier experiments at low magnetic fields $[13,14]$ and hints toward a nondivergent behavior at the CNP. This raises the question whether the $n_{\mathrm{el}}$-dependent renormalization of $v_{F}$ and thus the many-body effects are fundamentally different in the presence of LLs.

In this Letter, we report on extracting the renormalized LL energies and the corresponding $v_{F}$ as a function of $n_{\mathrm{el}}$ by optically probing gate-tunable magnetophonon resonances (MPRs) in suspended graphene. Magneto-Raman spectroscopy has successfully been used to probe inter-LL excitations in graphene $[19,21-31]$ and allows the study of the electron-phonon coupling and excitation lifetimes. Most importantly, this technique offers a suitable energy scale for measuring the $B$-field-tunable LL transition energies in the form of the Raman $G$ mode phonon energy ( $\approx 196 \mathrm{meV})$. Typically, such energy scales characteristic for LLs are difficult to reach by thermally activated transport, which is the method of choice for extracting the energy-band or $v_{F}$ renormalization at a negligible $B$ field [13].

To compare the renormalization effects at low and high $B$ fields, respectively, it is convenient to introduce an effective Fermi velocity $v_{F}$ for high $B$ fields, which captures the complete renormalization of the LL energies due to manybody effects [32]. Thus, the unique square root dependence of the LL spectrum of massless Dirac fermions has to be modified with a renormalized effective Fermi velocity $v_{F}$, which now depends on $B, n_{\mathrm{el}}$, and the LL index $\pm n$. The LL spectrum including many-body interactions thus reads $\varepsilon_{ \pm n}= \pm v_{F, n}\left(B, n_{\mathrm{el}}\right) \sqrt{2 e \hbar B n}$. Most interestingly, our study of magnetophonon resonances as a function of $n_{\mathrm{el}}$ reveals that $v_{F}$ does not scale logarithmically with $n_{\mathrm{el}}$, as is the case for $B \approx 0 \mathrm{~T}$, but rather shows a linear and thus finite dependence. We attribute this change in behavior to the suppression of the long-range Coulomb interaction for distances larger than the magnetic length $l_{B}=\sqrt{\hbar /(e B)}$. Moreover, we present a quantitative description of the evolution of $v_{F}$ in the presence of LLs within a tightbinding model [33] on the level of the Hartree-Fock approximation, finding a near-perfect agreement with our measurements. 
For our experimental study, we use a suspended graphene device offering high carrier mobility, low intrinsic strain, low charge carrier density inhomogeneity, and electron-electron interactions that are not screened by the environment. The device consists of an exfoliated graphene flake on a $\mathrm{Si} / \mathrm{SiO}_{2}$ substrate, contacted with $\mathrm{Cr} / \mathrm{Au}$ contacts and suspended by etching away $\approx 160 \mathrm{~nm}$ of $\mathrm{SiO}_{2}$. A subsequent current annealing step [34] gives rise to a carrier mobility exceeding $400000 \mathrm{~cm}^{2}$ (Vs) and a charge inhomogeneity of less than $n^{*} \approx 10^{9} \mathrm{~cm}^{-2}$ (see Supplemental Material [35]), which allow the observation of MPRs below $4 \mathrm{~T}$ [22-30]. The Si back gate permits the tuning of the charge carrier density. For the magneto-Raman measurements, we utilize a confocal, low-temperature micro-Raman setup, which permits combined optical and transport experiments and is equipped with a superconducting magnet. We use linearly polarized laser light $(\lambda=532 \mathrm{~nm})$ with a power of $0.5 \mathrm{~mW}$ and a spot size of $\approx 500 \mathrm{~nm}$. The scattered light is detected by a CCD spectrometer with a grating of 1200 lines $/ \mathrm{mm}$. All measurements were performed at a temperature of $4.2 \mathrm{~K}$.

To study magnetophonon resonances as a function of the charge carrier density, we vary $n_{\mathrm{el}}=\alpha\left(V_{g}-V_{0}\right)$ by tuning the applied gate voltage $V_{g}$, where $V_{0}=-0.2 \mathrm{~V}$ accounts for the residual doping. We extract the lever arm $\alpha=$ $3.15 \times 10^{10} \mathrm{~cm}^{-2} \mathrm{~V}^{-1}$ from a Landau fan measurement (see Supplemental Material [35]). For each specific $V_{g}$, we sweep $B$ from 0 to $6 \mathrm{~T}$ while recording Raman spectra. To study the coupling of the electronic system to the Ramanactive $E_{2 g}$ mode, we extract the position $\omega_{G}$ and width $\Gamma_{G}$ of the Raman $G$ peak by fitting a single Lorentzian. Their evolution as a function of the $B$ field for different values of $V_{g}$ is shown in Figs. 1(a) and 1(b), respectively. We observe the resonant coupling of the Raman $G$ mode phonon [22-28] to electronic transitions when its bare energy $\varepsilon_{\mathrm{ph}}=$ $\hbar \omega_{G}\left(B=0 \mathrm{~T}, n_{\mathrm{el}}=0 \mathrm{~cm}^{-2}\right) \equiv \hbar \omega_{0}$ matches the energy of a transition between the discrete LLs. Most prominently, this coupling results in a decrease of the phonon lifetime due to the excitation of electron-hole pairs, which results in an increased width $\Gamma_{G}$ of the Raman $G$ peak at resonance. To first order in the perturbation theory, the $E_{2 g}$ phonon couples only to LL excitations with $\Delta n= \pm 1[29,30]$. We thus focus on the coupling to LL transitions whose energies are given by $T_{n}=\varepsilon_{n+1}-\varepsilon_{-n}$ [see Figs. 1(c) and 1(d)]. Note that the influence of excitonic effects on $T_{n}$ is neglected here and will be discussed later in this Letter. The resonance condition $\varepsilon_{\mathrm{ph}}=T_{n}$ can be expressed as

$$
\hbar \omega_{0}=v_{F, T_{n}}\left(B_{T_{n}}, n_{\mathrm{el}}\right) \sqrt{2 e \hbar B_{T_{n}}}(\sqrt{n+1}+\sqrt{n}),
$$

where we define an effective Fermi velocity $v_{F, T_{n}}$ of the transition $T_{n}$ via $v_{F, T_{n}} \equiv T_{n} /[\sqrt{2 e \hbar B}(\sqrt{n+1}+\sqrt{n})]$ [41]. By measuring $\omega_{0}$ and the value of the $B$ field at which the resonance occurs, $B_{T_{n}}$, we can thus extract the
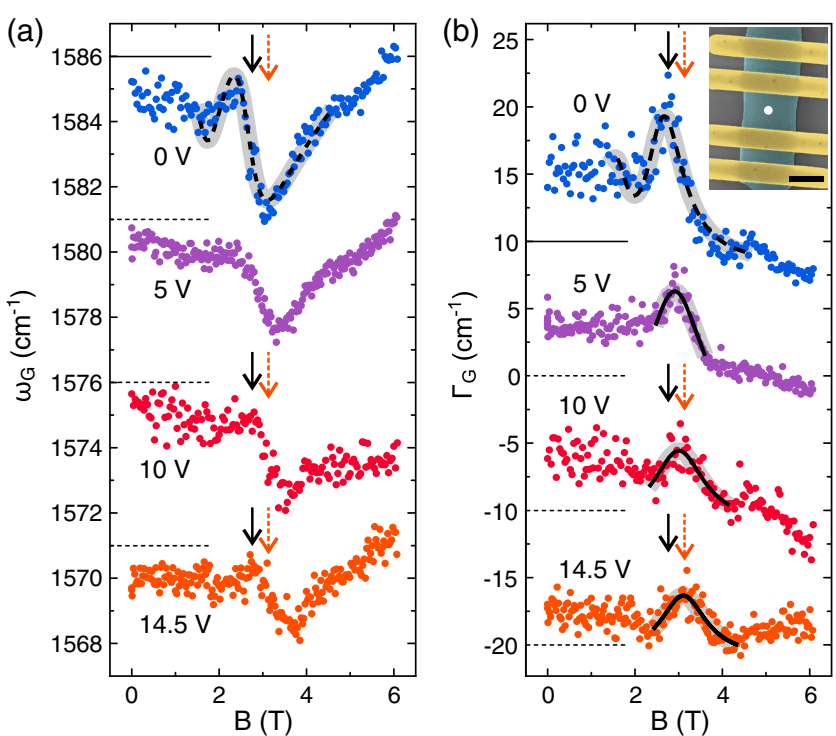

(c)

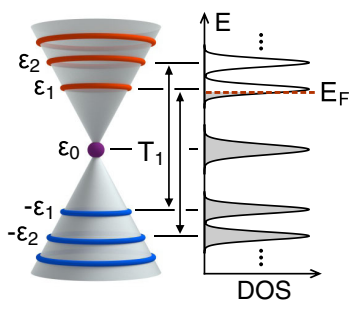

(d)

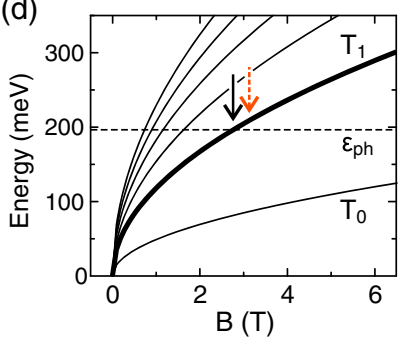

FIG. 1. (a), (b) Position and width of the Raman $G$ peak as a function of the $B$ field and gate voltage (graphs offset for clarity; compare horizontal dashed lines). Black and orange arrows highlight the $T_{1}$-MPR positions for $V_{g}=0$ and $14.5 \mathrm{~V}$, respectively. Dashed black curves represent our theoretical calculation [35], and full black lines depict Lorentzian fits to $\Gamma_{G}$ near the $T_{1}$-MPR. Inset: False-color scanning electron micrograph of the measured device. The white dot represents the laser spot, and the scale bar is $2 \mu \mathrm{m}$. (c) Illustration of the LL transition energies and density of states near $E_{F}$. (d) Full lines: LL transition energies for $v_{F}=1.35 \times 10^{6} \mathrm{~m} / \mathrm{s}$. Dashed line: Phonon energy at $B=0 \mathrm{~T}$.

effective Fermi velocity $v_{F, T_{n}}\left(B=B_{T_{n}}, n_{\mathrm{el}}\right)$. The experimentally determined $v_{F, T_{n}}$ evidently contains all corrections from electron-electron interactions. Hence, the position of the MPR provides a direct probe of the renormalized transition energy, parametrized by $v_{F, T_{n}}$. In particular, we are able to probe the charge carrier density dependence of $v_{F, T_{n}}$ by varying $V_{g}$.

In the following, we focus on the charge carrier density dependence of the $T_{1}$ transition [28-30], which gives rise to a resonance at $B_{T_{1}} \approx 3 \mathrm{~T}$ [Figs. 1(a) and 1(b)]. Increasing $\left|n_{\mathrm{el}}\right|$ leads both to an increase of $B_{T_{1}}$ [compare black and red arrows in Figs. 1(a) and 1(b)] and to a decrease of the strength of the $T_{1}$-MPR, which we define as the maximum value of $\Gamma_{G}$ at the resonance $B_{T_{1}}, \Gamma_{G, T_{1}}^{\max }$. For a more quantitative analysis, we fit single Lorentzians to $\Gamma_{G}$ [Fig. 1(b)] as a function of $B$ around the $T_{1}$-MPR to obtain $\Gamma_{G, T_{1}}^{\max }$ and $B_{T_{1}}$ [see Figs. 2(a) and 2(b)]. The observed 

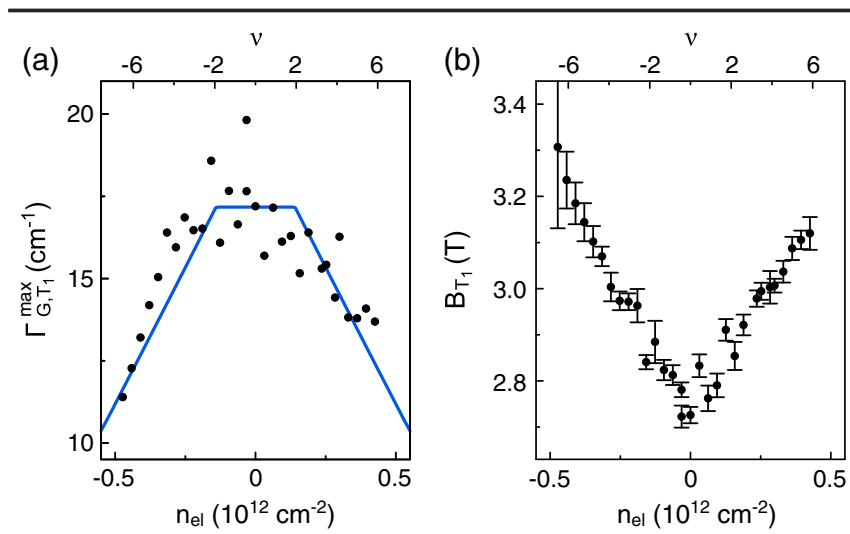

FIG. 2. (a) $G$ peak width at the $T_{1}$-MPR and (b) position of the $T_{1}$-MPR as a function of $n_{\mathrm{el}}$. Blue line: Theoretical calculation [35] with the average parameters from all measurements. Upper axis: Filling factor $\nu=n_{\mathrm{el}} h /(e B)$ at $B=3 \mathrm{~T}$.

behavior of $\Gamma_{G, T_{1}}^{\max }$ with $n_{\mathrm{el}}$ can be understood in terms of the increasing filling of different LLs and the resulting Pauli blocking. For small $\left|n_{\mathrm{el}}\right|$, the Fermi energy stays within the zeroth LL and, hence, $\Gamma_{G, T_{1}}^{\max }$ remains constant, as the $T_{1}$ transition involves only the transitions $-1 \rightarrow+2$ and $-2 \rightarrow+1$. For higher values of $\left|n_{\mathrm{el}}\right|$, the states belonging to the first LL are filled, and more and more of the degenerate LL transitions become blocked. The decrease of $\Gamma_{G, T_{1}}^{\mathrm{max}}$ with $\left|n_{\mathrm{el}}\right|$ is in good agreement with the theoretical prediction [blue line in Fig. 2(a); also see Supplemental Material [35]], while the linear scaling results from the linear filling of the LLs with $n_{\mathrm{el}}$.

Next, we analyze the charge carrier density dependence of the position $B_{T_{1}}$ of the $T_{1}$-MPR [see Fig. 2(b)]. According to Eq. (1), $B_{T_{1}}$ depends only on the value of the phonon frequency $\omega_{0}$ and on $v_{F, T_{1}}$. We rule out changes of $\omega_{0}$ due to tensile strain from electrostatically pulling the graphene flake as the origin of the observed shift in $B_{T_{1}}$, since the observed variation of $\omega_{0}$ is negligible (less than $2 \mathrm{~cm}^{-1}$; see Supplemental Material [35]). Furthermore, tensile strain would soften $\omega_{0}$; i.e., it would lead to a decrease of $B_{T_{1}}$ with increasing $n_{\mathrm{el}}$. Thus, the shift of $B_{T_{1}}$ can be caused only by a change of the LL excitation energies, as described by an $n_{\mathrm{el}}$-dependent effective Fermi velocity $v_{F, T_{1}}$. For a quantitative analysis of $v_{F, T_{1}}\left(n_{\mathrm{el}}\right)$, we employ Eq. (1) and the extracted $B_{T_{1}}$ to calculate $v_{F}$ as a function of $n_{\mathrm{el}}$ (see Fig. 3). We obtain an effective Fermi velocity ranging from $v_{F, T_{1}} \approx 1.36 \times 10^{6} \mathrm{~m} / \mathrm{s}$ close to the charge neutrality point to $v_{F, T_{1}} \approx 1.24 \times 10^{6} \mathrm{~m} / \mathrm{s}$ at $\left|n_{\mathrm{el}}\right|=0.4 \times 10^{12} \mathrm{~cm}^{-2}$. Most interestingly, we do not observe a logarithmically divergent behavior close to the $\mathrm{CNP}$, as is the case in the low $B$ field regime (see the inset in Fig. 3) $[13,14]$. Instead, we find a finite, linear decrease of $v_{F, T_{1}}$ as a function of $\left|n_{\mathrm{el}}\right|$. We attribute this linear behavior to the degeneracy of the states within one LL. Because of the degeneracy, the contribution of a certain LL to the

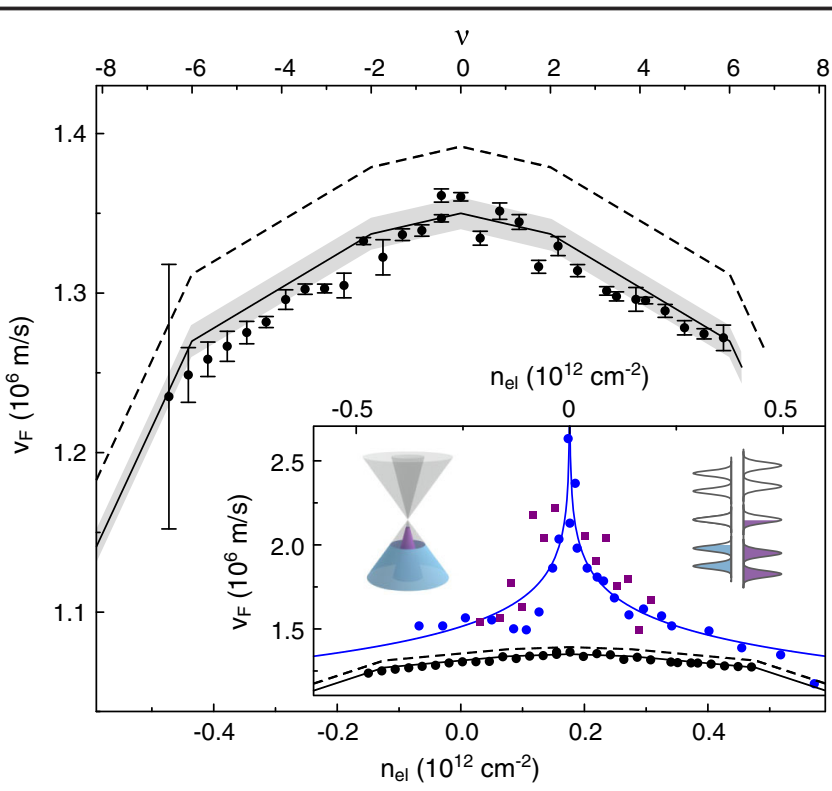

FIG. 3. Effective Fermi velocity as a function of $n_{\mathrm{el}}$. Solid (dashed) line: Calculation without (with) considering the excitonic binding energy. Gray-shaded area: Uncertainty due to $B$-field-dependent renormalization $[19,42]$. Upper axis: Filling factor $\nu$ at $B=3 \mathrm{~T}$. Inset: $v_{F}$ in the presence of LLs (black dots and lines as in main panel) and at a low $B$ field (blue dots from Ref. [13], purple squares from $\mathrm{SdHO}$ measurements on our device). Blue line: Theoretical expectation at low $B$ field. Illustrations: Renormalization of the band structure with $n_{\mathrm{el}}$ at a low (left) and high (right) $B$ field.

renormalization of $v_{F, T_{1}}$ effectively equals the sum of the contributions of all its states weighted by the filling of the LL. Since the partial filling factor scales linearly with $n_{\mathrm{el}}$, so does the renormalization of $v_{F, T_{1}}$, as long as $n_{\mathrm{el}}$ is varied in a range for which the Fermi level $E_{F}$ stays within a single LL. When $E_{F}$ enters a different LL, the slope of $v_{F, T_{1}}$ changes as the contribution of a different LL is now added.

We confirm this qualitative argument and the experimental observation by quantitative calculations on the level of the Hartree-Fock approximation (HFA) within a tightbinding model [33] (see Supplemental Material [35]). In the HFA, the single-particle LL energies are renormalized by contributions from all occupied states via the direct Coulomb (Hartree term) and exchange interactions (Fock term): $\varepsilon_{n}\left(B, n_{\mathrm{el}}\right)=\varepsilon_{n}^{0}(B)+\Sigma_{n}^{\mathrm{HF}}\left(B, n_{\mathrm{el}}\right)$, where $\varepsilon_{n}^{0}$ denotes the bare LL energies and

$$
\begin{aligned}
\sum_{n}^{\mathrm{HF}}\left(B, n_{\mathrm{el}}\right)= & \frac{1}{N_{m}} \sum_{m} \sum_{n, m}^{\mathrm{HF}}\left(B, n_{\mathrm{el}}\right) \\
= & \frac{1}{N_{m}} \sum_{m} \sum_{n^{\prime}, m^{\prime}} \bar{\nu}_{n^{\prime}}\left(B, n_{\mathrm{el}}\right) \\
& \times\left(2 v_{\substack{\left(n_{n}, m^{\prime} \\
\left(n^{\prime}, m^{\prime}\right)\right.}}^{\mathrm{Hart}}(B)-v_{\substack{(n, m) \\
\left(n^{\prime}, m^{\prime}\right)}}^{\mathrm{Fock}}(B)\right)
\end{aligned}
$$


is the self-energy of LL $n$, averaged over all $N_{m}$ degenerate states, labeled by the quantum number $m$ [43], and $v_{(n, m),\left(n^{\prime}, m^{\prime}\right)}^{\text {Hart }{ }^{\prime} \text {, }}(B)$ represent the direct Coulomb and exchange matrix elements, respectively, between the LL states $|n, m\rangle$ and $\left|n^{\prime}, m^{\prime}\right\rangle$. Finally, $\bar{\nu}_{n}\left(B, n_{\mathrm{el}}\right)=n_{\mathrm{el}} h /(4 e B)-n+1 / 2$ denotes the partial filling factor of LL $n$, which is set to 0 (1) for $\bar{\nu}_{n}<0(>1)$ and equals the occupancy of LL $n$. Including the Hartree-Fock correction, the $T_{n}$-transition energy reads

$$
T_{n}\left(B, n_{\mathrm{el}}\right)=\varepsilon_{n+1}^{0}-\varepsilon_{-n}^{0}+\Sigma_{n+1}^{\mathrm{HF}}-\Sigma_{-n}^{\mathrm{HF}} .
$$

To account for the intrinsic screening of the graphene sheet, we use an effective dielectric constant of $\epsilon=3.1$ to screen all Coulomb matrix elements by an additional factor of $1 / \epsilon$, in agreement with earlier work [13]. Expressing $T_{n}\left(B, n_{\mathrm{el}}\right)$ in terms of the effective Fermi velocity $v_{F, T_{n}}$ [compare Eq. (1)], Eq. (3) implies

$v_{F, T_{n}}\left(n_{\mathrm{el}}\right)=v_{F, T_{n}}\left(n_{\mathrm{el}}^{0}\right)+\frac{\Delta \Sigma_{n+1}^{\mathrm{HF}}\left(n_{\mathrm{el}}\right)-\Delta \Sigma_{-n}^{\mathrm{HF}}\left(n_{\mathrm{el}}\right)}{\sqrt{2 e \hbar B_{T_{n}}}(\sqrt{n+1}+\sqrt{n})}$,

where $\Delta \Sigma_{n}^{\mathrm{HF}}\left(n_{\mathrm{el}}\right)=\Sigma_{n}^{\mathrm{HF}}\left(n_{\mathrm{el}}\right)-\Sigma_{n}^{\mathrm{HF}}\left(n_{\mathrm{el}}^{0}\right)$ denotes the difference in self-energies and $n_{\mathrm{el}}^{0}=0 \mathrm{~cm}^{-2}$. Note that, in this difference, all contributions from states outside the energy window defined by $n_{\mathrm{el}}^{0}$ and $n_{\mathrm{el}}$ drop out for a constant $B$ field, as their occupancies do not change. This applies, in particular, to contributions from states deep inside the valence band, beyond the UV cutoff in renormalization group approaches $[9,10,13]$. These states influence only the overall scale of $v_{F}$, represented by $v_{F, T_{1}}\left(n_{\mathrm{el}}^{0}\right)$. We experimentally extract $v_{F, T_{1}}\left(n_{\mathrm{el}}^{0}\right)=1.35 \times 10^{6} \mathrm{~m} / \mathrm{s}$ and use it as input for our calculation. As seen in Fig. 3, our calculation predicts a piecewise-linear $v_{F}\left(n_{\mathrm{el}}\right)$, which is in excellent agreement with our experimental results [42] and very recent theoretical work [44].

In order to compare $v_{F, T_{1}}$ with measurements of $v_{F}$ at low $B$ fields extracted by transport experiments $[13,14]$, it is important to discuss the so far neglected excitonic effects in our MPR analysis. As we probe electron-hole pair excitations, the experimentally extracted LL transition energies $T_{n}=\varepsilon_{n+1}-\varepsilon_{-n}+\varepsilon_{n+1,-n}^{\text {bind }}$ include a (negative) binding energy of the electron-hole pair $\varepsilon_{n+1,-n}^{\text {bind }}$. Consequently, our experimentally extracted $v_{F, T_{1}}\left(n_{\mathrm{el}}^{0}\right)$ already contains an excitonic component of $\delta v_{F, T_{1}}^{\text {bind }}=$ $\varepsilon_{2,-1}^{\text {bind }} /[\sqrt{2 e \hbar B}(\sqrt{2}+1)]$ [compare Eq. (1)]. To correct for the excitonic effects and thus permit a sensible comparison to the $v_{F}$ extracted from transport measurements, we estimate $\varepsilon_{n+1,-n}^{\text {bind }}$ by approximating it as the difference of the direct and exchange Coulomb matrix elements, averaged over all possible pairs of degenerate LL states:

$$
\varepsilon_{n+1,-n}^{\mathrm{bind}}=1 / N_{m}^{2} \sum_{m, m^{\prime}}\left(v_{\substack{(n+1, m),\left(-n, m^{\prime}\right)}}^{\mathrm{Hart}}-v_{\substack{(n+1, m) \\\left(-n, m^{\prime}\right)}}^{\mathrm{Fock}}\right) .
$$

The numerical evaluation of this expression yields an $n_{\mathrm{el}}$-independent estimate of $\varepsilon_{2,-1}^{\mathrm{bind}} \approx-6 \mathrm{meV}$, when including the screening factor of $1 / \epsilon$. When correcting $v_{F, T_{1}}$ for $\delta v_{F, T_{1}}^{\text {bind }}$, we obtain values for the effective Fermi velocity without any excitonic effects, as shown as the black dashed line in Fig. 3.

The inset in Fig. 3 shows a comparison of $v_{F}$ for low magnetic fields $(B<0.5 \mathrm{~T})$ and in the presence of LLs $(\approx 3 \mathrm{~T})$. The purple squares represent $v_{F}\left(n_{\mathrm{el}}\right)$ at low $B$ fields, extracted from temperature-dependent Shubnikov-de Haas oscillation ( $\mathrm{SdHO}$ ) measurements (see Supplemental Material [35]) taken on the same device used for our MPR study. They are in good agreement with the logarithmic behavior of $v_{F}$ at low $B$ fields reported by Elias et al. [13] (blue dots and line). Most interestingly, there is a striking difference in the $n_{\mathrm{el}}$ dependence between $v_{F}$ extracted at low $B$ fields from transport experiments and $v_{F, T_{1}}$ determined at high $B$ fields from our optical measurements. Note that the magneto-Raman measurements always probe $v_{F}$ away from the Dirac point at approximately half the phonon energy $(\approx 100 \mathrm{meV})$, while transport experiments extract the band slope at the Fermi surface. However, previous ARPES [16] and scanning tunneling spectroscopy [17] studies established that the renormalized bands remain linear within an energy window around the CNP of at least $200 \mathrm{meV}$ (see the left schematic in the inset in Fig. 3). As we both probe $v_{F}$ and tune the Fermi energy within this energy window, the exact energy at which $v_{F}$ is probed is irrelevant. Consequently, it is justified to compare our results to the ones from transport measurements at low $B$ fields. Since the excitonic correction cannot account for the change in effective Fermi velocities, we conclude that the difference in $v_{F}$ is not due to the way in which $v_{F}$ is determined but rather due to the difference in electron-electron interaction at low $B$ fields and in the presence of LLs. At low $B$ fields, the selfenergy correction to $v_{F}$ diverges due to the long-range behavior of the Coulomb interaction and the delocalized nature of the Dirac electrons at the $K$ point. By contrast, high magnetic fields exponentially localize the electronic wave functions once LLs are present, with a decay constant on the order of the magnetic length $l_{B}$ (see Supplemental Material [35]). As a result, the long-range divergence is eliminated.

In conclusion, we studied the charge carrier density dependence of the effective Fermi velocity in the presence of LLs by studying magnetophonon resonances. In contrast to the logarithmic renormalization of $v_{F}$ at low magnetic fields, we find that $v_{F}$ remains finite in the LL regime. While the suppression of the divergence of the exchange self-energy can be traced back to the confinement of the 
electron wave function in a magnetic field, the observed linear scaling of $v_{F}$ with $n_{\mathrm{el}}$ can be attributed to the degeneracy of the LLs. Tight-binding calculations quantitatively verify our experimental findings, confirming that electron-electron interactions can indeed change dramatically for different magnetic field regimes. The general insight into the influence of many-body effects on electronic excitation in strong magnetic fields gained from our findings makes them also applicable to the study of other low-dimensional materials and can be of great value for the effective tuning of material properties.

We thank C. Neumann, E. Andrei, and F. Guinea for helpful discussions and M. Goldsche for help with sample fabrication. Support by the (EU)European Research Council (Grant No. 280140), the Helmholtz Nano Facility [45], the Deutsche Forschungsgesellschaft, and the European Union's Horizon 2020 program under Grant Agreement No. 696656 (Graphene Flagship) are gratefully acknowledged. S. R. and L. W. acknowledge financial support by the National Research Fund (FNR) Luxembourg (projects RAMGRASEA and TMD-nano: INTER/ANR/13/20/NANOTMD). F. L. acknowledges financial support by the FWF (SFB-F41 ViCoM) and numerical support by the Vienna Scientific Cluster (VSC).

*sonntag@physik.rwth-aachen.de

[1] V. N. Kotov, B. Uchoa, V. M. Pereira, F. Guinea, and A. H. Castro Neto, Rev. Mod. Phys. 84, 1067 (2012).

[2] C. Dean, A. Young, P. Cadden-zimansky, L. Wang, H. Ren, K. Watanabe, T. Taniguchi, P. Kim, J. Hone, and K. Shepard, Nat. Phys. 7, 693 (2011).

[3] A. Young, C. Dean, L. Wang, H. Ren, P. Cadden-Zimansky, K. Watanabe, T. Taniguchi, J. Hone, K. Shepard, and P. Kim, Nat. Phys. 8, 550 (2012).

[4] A. N. Grigorenko, M. Polini, and K. S. Novoselov, Nat. Photonics 6, 749 (2012).

[5] M. B. Lundeberg, Y. Gao, R. Asgari, C. Tan, B. Van Duppen, M. Autore, P. Alonso-González, A. Woessner, K. Watanabe, T. Taniguchi, R. Hillenbrand, J. Hone, M. Polini, and F. H. L. Koppens, Science 357, 187 (2017).

[6] D. N. Basov, M. M. Fogler, and F. J. García de Abajo, Science 354, aag1992 (2016).

[7] D. Bandurin, I. Torre, R. K. Kumar, M. B. Shalom, A. Tomadin, A. Principi, G. Auton, E. Khestanova, K. Novoselov, I. Grigorieva, L. Ponomarenko, A. Geim, and M. Polini, Science 351, 1055 (2016).

[8] J. Crossno, J. K. Shi, K. Wang, X. Liu, A. Harzheim, A. Lucas, S. Sachdev, P. Kim, T. Taniguchi, K. Watanabe, T. A. Ohki, and K. C. Fong, Science 351, 1058 (2016).

[9] J. González, F. Guinea, and M. Vozmediano, Nucl. Phys. B424, 595 (1994).

[10] J. González, F. Guinea, and M. A. H. Vozmediano, Phys. Rev. B 59, R2474 (1999).

[11] T. Stauber, P. Parida, M. Trushin, M. V. Ulybyshev, D. L. Boyda, and J. Schliemann, Phys. Rev. Lett. 118, 266801 (2017).
[12] S. Das Sarma and E. H. Hwang, Phys. Rev. B 87, 045425 (2013).

[13] D. Elias, R. Gorbachev, A. Mayorov, S. Morozov, A. Zhukov, P. Blake, L. P. I. Grigorieva, K. Novoselov, F. Guinea, and A. Geim, Nat. Phys. 7, 701 (2011).

[14] G. Yu, R. Jalil, B. Belle, A. S. Mayorov, P. Blake, F. Schedin, S. V. Morozov, L. A. Ponomarenko, F. Chiappini, S. Wiedmann, U. Zeitler, M. Katsnelson, A. Geim, K. Novoselov, and D. Elias, Proc. Natl. Acad. Sci. U.S.A. 110, 3282 (2013).

[15] A. Bostwick, F. Speck, T. Seyller, K. Horn, M. Polini, R. Asgari, A. H. MacDonald, and E. Rotenberg, Science 328, 999 (2010).

[16] D. A. Siegel, C.-H. Park, C. Hwang, J. Deslippe, A. V. Fedorov, S. G. Louie, and A. Lanzara, Proc. Natl. Acad. Sci. U.S.A. 108, 11365 (2011).

[17] J. Chae, S. Jung, A. F. Young, C. R. Dean, L. Wang, Y. Gao, K. Watanabe, T. Taniguchi, J. Hone, K. L. Shepard, P. Kim, N. B. Zhitenev, and J. A. Stroscio, Phys. Rev. Lett. 109, 116802 (2012).

[18] A. Luican, G. Li, and E. Y. Andrei, Phys. Rev. B 83, 041405 (2011).

[19] C. Faugeras, S. Berciaud, P. Leszczynski, Y. Henni, K. Nogajewski, M. Orlita, T. Taniguchi, K. Watanabe, C. Forsythe, P. Kim, R. Jalil, A. K. Geim, D. M. Basko, and M. Potemski, Phys. Rev. Lett. 114, 126804 (2015).

[20] A. A. Sokolik, A. D. Zabolotskiy, and Y. E. Lozovik, Phys. Rev. B 95, 125402 (2017).

[21] C. Faugeras, P. Kossacki, A. Nicolet, M. Orlita, M. Potemski, A. Mahmood, and D. Basko, New J. Phys. 14, 095007 (2012).

[22] C. Neumann, D. Halpaap, S. Reichardt, L. Banszerus, M. Schmitz, K. Watanabe, T. Taniguchi, B. Beschoten, and C. Stampfer, Appl. Phys. Lett. 107, 233105 (2015).

[23] J. Yan, S. Goler, T. D. Rhone, M. Han, R. He, P. Kim, V. Pellegrini, and A. Pinczuk, Phys. Rev. Lett. 105, 227401 (2010).

[24] C. Faugeras, M. Amado, P. Kossacki, M. Orlita, M. Kühne, A. A. L. Nicolet, Y. I. Latyshev, and M. Potemski, Phys. Rev. Lett. 107, 036807 (2011).

[25] S. Goler, J. Yan, V. Pellegrini, and A. Pinczuk, Solid State Commun. 152, 1289 (2012).

[26] C. Faugeras, M. Amado, P. Kossacki, M. Orlita, M. Sprinkle, C. Berger, W. A. de Heer, and M. Potemski, Phys. Rev. Lett. 103, 186803 (2009).

[27] X. Shen, C. Qiu, B. Cao, C. Cong, W. Yang, H. Wang, and T. Yu, Nano Res. 8, 1139 (2015).

[28] C. Neumann, S. Reichardt, M. Drögeler, B. Terrés, K. Watanabe, T. Taniguchi, B. Beschoten, S. V. Rotkin, and C. Stampfer, Nano Lett. 15, 1547 (2015).

[29] T. Ando, J. Phys. Soc. Jpn. 76, 024712 (2007).

[30] M. O. Goerbig, J.-N. Fuchs, K. Kechedzhi, and V. I. Fal'ko, Phys. Rev. Lett. 99, 087402 (2007).

[31] C. Faugeras, M. Orlita, and M. Potemski, J. Raman Spectrosc. 49, 146 (2018).

[32] Note that in this context the effective Fermi velocity $v_{F}=$ $v_{F, n}\left(B, n_{\mathrm{el}}\right)$ is no longer linked to its definition as the slope of the energy bands but rather directly describes the renormalization of the LL energies. 
[33] L. A. Chizhova, J. Burgdörfer, and F. Libisch, Phys. Rev. B 92, 125411 (2015).

[34] K. I. Bolotin, K. Sikes, Z. Jiang, M. Klima, G. Fudenberg, J. Hone, P. Kim, and H. Stormer, Solid State Commun. 146, 351 (2008).

[35] See Supplemental Material at http://link.aps.org/ supplemental/10.1103/PhysRevLett.120.187701 for additional details on the tight-binding calculations, the model used to fit the MPRs, more detailed electrical characterizations, and Refs. [36-40].

[36] C. Lanczos, J. Res. Natl. Bur. Stand. 45, 255 (1950).

[37] S. G. Sharapov, V. P. Gusynin, and H. Beck, Phys. Rev. B 69, 075104 (2004).

[38] M. Katsnelson, Graphene: Carbon in Two Dimensions (Cambridge University Press, Cambridge, England, 2012).

[39] N. Y. Astrakhantsev, V. V. Braguta, and M. I. Katsnelson, Phys. Rev. B 92, 245105 (2015).

[40] V. P. Gusynin, V. A. Miransky, and I. A. Shovkovy, Phys. Rev. D 52, 4718 (1995).

[41] In terms of the renormalized Fermi velocities $v_{F, n+1}$ and $v_{F,-n}$ of the respective Landau levels, the effective
Fermi velocity of the $T_{n}$ transition reads $v_{F, T_{n}}=$ $\left(v_{F, n+1} \sqrt{n+1}+v_{F,-n} \sqrt{n}\right) /(\sqrt{n+1}+\sqrt{n})$.

[42] Note that we experimentally extract $v_{F, T_{1}}$ at slightly different $B$ fields for each value of $n_{\mathrm{el}}$, due to the $n_{\mathrm{el}}$-dependent renormalization of $v_{F, T_{1}}$. As shown previously [19], the effective Fermi velocity also exhibits a $B$-field-dependent renormalization proportional to $\log \left(B_{2} / B_{1}\right)$. However, the magnitude of this renormalization $\delta v_{F} \approx 0.02 \times 10^{6} \mathrm{~m} / \mathrm{s}$ for the observed range of $B_{T_{1}}$ values $(2.8-3.2 \mathrm{~T})$ is small compared to the $n_{\mathrm{el}}$-induced corrections, and hence we neglect it.

[43] The physical meaning of $m$ depends on the choice of gauge for the vector potential. In the Landau gauge, $m$ represents the momentum in the $y$ direction, while in the symmetric gauge, $m$ represents the $z$ component of the angular momentum.

[44] A. A. Sokolik and Y. E. Lozovik, Phys. Rev. B 97, 075416 (2018).

[45] Forschungszentrum Jülich GmbH, J. Large-Scale Res. Facilities 3, A112 (2017). 